\title{
Waiting for Marcel Orphüls: The Literature of Authoritarian Rule and Resistance in Latin America
}

David M. K. Sheinin

\section{(2) OpenEdition \\ Journals}

Electronic version

URL: https://journals.openedition.org/temoigner/1043

DOI: $10.4000 /$ temoigner. 1043

ISSN: 2506-6390

Publisher:

Éditions du Centre d'études et de documentation Mémoire d'Auschwitz, Éditions Kimé

\section{Printed version}

Date of publication: 1 October 2014

Number of pages: 190-194

ISBN: 978-2-84174-674-3

ISSN: 2031-4183

Electronic reference

David M. K. Sheinin, "Waiting for Marcel Orphüls: The Literature of Authoritarian Rule and Resistance in Latin America", Témoigner. Entre histoire et mémoire [Online], 118 | 2014, Online since 01 October 2015, connection on 03 February 2022. URL: http://journals.openedition.org/temoigner/1043 ; DOI: https://doi.org/10.4000/temoigner.1043 


\section{$\checkmark$ Librairie}

\section{SECRET D'ENFANCE}

Jean-Claude Snyders
Paris, Le Manuscrit, 2012, 317 p.

enouveau livre de Jean-Claude Snyders prend forme d'un récit. Sur le mode d'une parabole, s'isole dans un paysage, dans des habitudes, dans ses pensées, dans son silence. Bref, il tait sa souffrance. Peut-être lui est-il arrivé des choses si sombres qu' s'abstient de les considérer: «Y Ya-t-il un seul être dont souhaiterait la proximité? », se demande alors l'auteu du livre, frère de centaines de milliers dautres dontles parents ont été déportés dans des camps par les nazis. Curieusement, l'émotion que pourrait éprouver ( étranger, devient pour lui une faiblesse, tout soutien laffection une, pour ne paslappeler une ruse. Même lafection de son enfant lui hinspire de la méflance. Elle risque de le faire changer, de briser son isolement, son refuge. Cet honne, cest, pour ainsi dire, le fantốme de Georges Snyders, le père de lauteur, déporté

Ce livre pourrait rester un témoignage, celui de ces enfants confrontés au silence de leurs parents sur leur sejour dans les canps de déportation et dextermination nazis. Mais il est bien plus que cela, car il a valeur dintrospection, de plongée en soi-mêne et, a ce lenfance. lenfance. L'enabilité, un premier « péché '́ai-je fait, se dilté, un premier "péché » : quel mal du père : se disque le mal est en moi, même si jence du père: " puisque le mal est en moi, même si je m'y efforce je ne peux pas cesser detre mauvais » [p. 48] Ce fils porte aussi en lui une mission. Celle de vele Ce fils porte aussien lui une mission. Celle de venir à lié, blessé, détruit.

La scène se renverse, plus tard, lorsque l'enfant, devenu père à son tour, est entouré de ses propres nfants et quà leur contact, il repense à cette parti de sa vie. Ce n'est déjà plus sa propre enfance qu’il revisite, mais l'enfance en soi. Ce lieu des espoirs et des désespoirs, des amours et des haines, des désirs et des interdictions, des larmes et des rires. Jean-Claude Snyders développe ainsi, à partir de cette grande souffrance incompréhensible pour lui, une réflexion sur la genèse des passions d'amour et de haine, d'affection et de détestation. Il les tire des plus petites expériences de la vie quotidienne ou des plus grands événement de Phistoire : «Les choses hideuses que j’ai perçue jadis, écrit-il, qui sont entrees en moi, m'ont convaincu de mon horreur profonde; depuis longtemps j'essaie

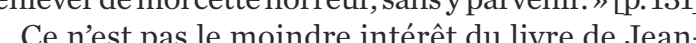
Ce n'est pas le moindre intérét du livre de JeanClaude Snyders que de décrire le chemin qui conduit de ces terribles souvenirs à une forme de dépassement, peut-être de résurection, chemin callouteux, dans des épsage austere, exposé aux vents et aux rafales

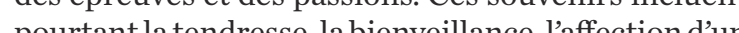
pourtant latendresse, labienveillance,l'affection d'un père pour son enfant. Avec, encreux dans ce message, tout ce qui a manqué à dau

On l'aura compris, le livre de Jean-Claude Snyders se situe au carrefour des Memoires, de la psychologie et de la philosophie de léducation. Mais au plus profon. de lui-même, il est surtout une remontee vers l'espoi pour tous ceux et toutes celles qui ont souffert de la souffrance que les parents leur ont léguee. Le livre se termine sur un texte de Georges Snyders, perre de de la libération du Le Monde pour le cinquantenaire etémoération du canp d'Auschwitz. Texte terrifiant André Rauch

\section{AURAIS-JE ÉTÉ RÉSISTANT \\ OU BOURREAU?}

Pierre Bayard

Paris, Éditions de Minuit, « Paradoxe », 2013, 158 p.

a question qui fait titre en a hanté plus d'un. Dan sa formelle généralité, celle-ci est privée de toute Pierre Bayard la refuse et se l'adresse très directement.
C'est elle et elle seule que son livre examine. Le proogue en guide la lecture.

(1) pessonnage dont la fonction est de le représenter lui, Pierre Bayard, tel qu'il se transporte, non une généra(ix encén d'entrée à l'́́cole nor co d'Ul dentré à lEcole normale superieure de la rue d Ulm à Paris. Cette transposition et ce déplacement réclament celui que Bayard appelle le personnage-délégué.

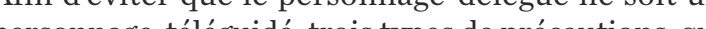
personage-telécuide, trois types de précautions, qu sont autant de protections pour lauteur, sont prises : sermior des lois scientifiques du comportement (behaviorisme, expérience de Milgran et sa critique),

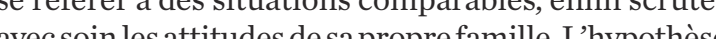
avec soinles attitudesdesaproprén se confhypothè exige que le personnage-delégué se confronte a des des personnes réelles. Elle se conforte rup tres es peron tions vraies auxquelles se plait la fiction que déploie d'un batilon allen d'un batallon allemand qui, en juillet 1942, refusent lordre de commettre le meurtre de masse. Le personnage-délégú reçoitunesortede corporeltépsychique lauteur quion de "personnalté potentielle » que the Piecre à linconscient.

Pierre Bayard inscrit sa démarche et son livre dan un procès defiction. Iltientaunombre de ses mérites pouvoir d approcher une vérite. Comment alors lire ce . D' ó ar Dieu. Prescriptions ou recommandations dext sur Dieu. Prescriptions ou recommandations dont se voit pour le lecteur qui, le livre acheve, acceptera, nous semble-t-il, plus aisément que le texte porte sur Dreu que sur la léc par l'auteur de Comment parler livres quel'on 'u as lus ?N'est de Commer iivesquelon ápas lus? Nest pas che sorte dimpert nente faussepiste? Tentons, pour notre part, de parler un live que nous avons ly.

La question à laquelle le livre se propose de repondre prend consistance en 1940, quand Pétain devient le chef dune France en partie occupée par les troupes et les services de IAllemagne nazie. Que faire? puisser la defaite, accéter la collaboration avec au contraire, s'y opposer, mais alors de quelle façon. Le chroniqueur découvre qu'il est difficile de s'éloigner de a chronologie du livre qui commence par une analys Jucien. On se souvient que Lucien Lacombe est un jeste trois Dans les quatre parties quiorganisentlensemble, rois ordres sont repérables : un ordre conceptuel qu oique, empathie, peur, de soi). À cet ordre concepluel co, enpant tuel correspond un choix, se présente une décision ou

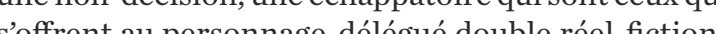
s'our d'université d'aujourd'hu Choix décision, expeur d'université daujourd hul. Choix individus écls que l'an-choixs'adossent alors à des individus réels que lauteur convoque, auxquels il se (Belon

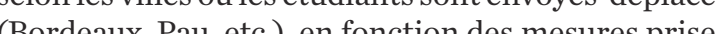
(Bor le ré  gnement, mise en place du STO), apparaissent dan réels : Daniel Cordier, futur secrétaire de Jean Mouhin : Daniel Cordier, futur secrétaire de Jean Moude ; petite ville de Ch Gonctà venir; Les Justes peur Trocmé ct fa femme S Lousa Mendes, consu du Portugal à Bordeaux qui désobéissant as, consul nement de Salazar sione sans compter des visas a que les Juifs et tou signe sans conpter des visas afin

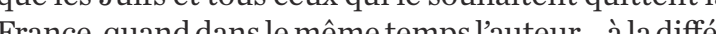
ré dirigé par l'historien vichyste Carcopino supérieure jours des Ihistorienvichysté Carcopino. Ce sont toulaus deur appelle ̀̀ lui, telle Milena Jesenka qui tie lau sonnelités camp de Ravensbrick. Comment ces perleur choix rendre compte des comportent expliquer ment la peur du risque physique masque le motif vra de ne reur faire ou à linverique masque le motif vra fait convan convaincre qu'on mène une action de résistance ? Ce ne sont pas les mesures anti-juives du régime de Vichy qui pousseront le personnage-délégué a s'engager tarhom con Une for soucto

Une fois soupçonnées et invalidées les bonnes raisons dêtre bonnes, cest-à-direles raisons commandées parlidéologie, lappartenance à un groupe, lempathie, 
autant être capable de s'engager ou de s'approcher de action, on atteint ce que la dernière partie du livr cherche à cerner, «le point de bascule ». Le point ce che détermo politique, lethique ne lont pas enporté pour se tét termes: soi-menc, les autres (et non autri,

Il faut sans doute être psychanalyste pour faire hypothèse de cette personnalité potentielle dont le sujet conscient náa ni dée, nireprésentation, ni anticipation, et sans doute pas le désir chenisager ni de savoir

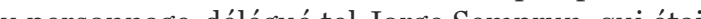

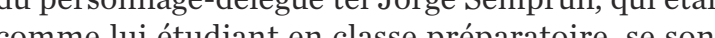
commé lui étudiant en classe préparatoire, se sont de nous, a co con

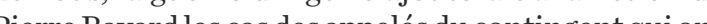
lofú comme Noël Favrelière d'abattre le prison on re cour arabe qu'on leur confint

On se demande quel est ce Dieu, qu’on dirait volontiers athée, dont parle Pierre Bayard si ce n'est l'énigm quenous sommesànous-même. Il n'est pas indifferent, ou plutot pas surprenant, que ce soit un analyste qui été celui-ci ou celui-là, résistable réponse: aurai-je chéch que la procédure bâtie pour écrire le livre paraît l'emcrorive tion quile motive. Car li y a dans lentreprise de Bayard a lais prousse ctvaité. Au sens dungenre pictura bien connu, au sens où

Michel Enaudeau

\section{FUIR LE REICH, LES RÉFUGIÉS \\ JUIFS DE 1933 À 1946}

Deborah Dwork Robert Jan Van Pe

Paris, Calmann Lévy/Mémorial de la Shoah, 2012, 476 p.

e sujet du livre est le sort des Juifs qui sont parvenus à fuir l'Allemagne de 1933 à 1946. Contras'agit, le plus souvent, nullement du récit de destins de personnes rescapées, mais bien, pour reprendre la distinction de Primo Levi, de destins de personnes naudistinction de Primo Levi, de destins de personnesnau-

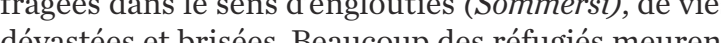

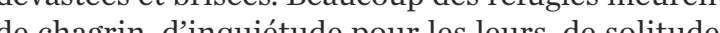
de chagrin, dinquiétude pour les leurs, de solitude. Duelques-uns, célebres ou inconnus, se suicident. Deborah Dwork et Robert Van Pelt décivent pleinement un aspect de la Shoah, malgré les apparence

Sans doute Fuir le Reich apprendra-t-il peu aux historiens. Mais pour le plus grand nombre des lecteurs les auteurs réunissent en un seul volume les aspects essentiels de cette histoire. Les differents moyen auxquels les nazis ont pensé pour chasser les Juifs au cours de la guerre sont soigneusement massacre au cours de la guerre sont soigneusement présentés. Reich tles réactions d'indifférence, d'impuis dans Remplicestét complicté et, parfois tout de mône, de live dance dans chacun des pays du reste du monde. Le livre contient ainsi un grand nombre de mises au point sur les polidu Royaciles de la Suisse. Quelques figures hérö̈ques soveétiqueou de la Suisse. Qu aussi tels le consul japonais en Lituanie, Chiune Sugihara, qui déliva aux Juifs des visas de transit pour le Japon ou Paul Grininger, ch réfugiés de far dentGall qui suisse qui donna aux réfugiés de faux papiers (ce quilu valut un córable jusqu'à sa moutsion de la police et une vie miserable jusquâ sa mort en 1972). cette habitante de Zurich qui organisa à partir de cette habitante de Zurich qui organisa à partir de sa juifs arde un véritable service postal entre les réfugié juifs allenands et leurs fanilles. Le lecteur trouvera egalement dans le live beaucoup d informations sur l'histoire de IOSE, l'ouvre de secours aux enfants. Lhistoire des espérances sionistes - quelques foi dęçues - et lhis leire de Un

Un grand nombre d'histoires personnelles des victimes, souvent très émouvantes, viennent illustrer régulièrement l'histoire générale, que ce soit la destine de poit la destinée dillo Stefan Zweig.

On voit ainsi, dans les premières années, la France accepter les réfugiés même dans la misère. Ce n'est pas le cas de lAngleterre qui prêfere "prendre les meil- ter. Les Juifs sont « comme les animaux qui, pour se protéger, changent de couleur et s’adaptent à celle la vegetation environnante. » On suit ainsi les proje à Maser Ju à Madagascar, dans la region de Lublin ou en Russie arctique. On accompagne les exilés dans tous les pays Sud et en Asie ju Sul Shan an Sud et en Asie jus sura Shangha. Le livre montre bien comes allemandes, refusent de se considerer comme une minorité et dêtre concernés par en faveur des «minorités » [p. 85].

En ce qui concerne la France, les passages sur $\mathrm{l}$ stuation des Juifs dans les camps d'internement sont accablants. Bien des récits sont déchirants comme celui

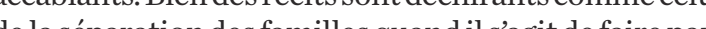
dela séparation des fanilles quandís agit de faire par-

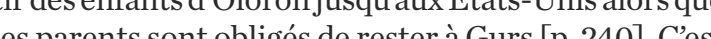
es par te sont obliges de resto à tir de drare des enfor juifs qui allaient enfin partir de Marseille vers les Etats-Unis, mais qui perdent Torch (débarquement des Américarès l Opération Torch (debarquenent des Anéricains et des Anglais Le Le livre ne sarrete pas avecla fin de la guerre etl'o

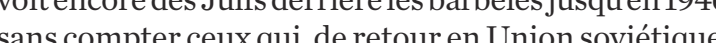
sans compter ceux qui, de retour en Union soviétique, y sont simplement exécutés (de même que beaucoup ćn général Patton traiter encore, la guerre finie, les Juif «despèce de sous-hommes», son collegue Eisenhowe ainsi dailleurs que le président Truman sont ré

Le livre sachive sur les diffecttes des survivan

Le livre s'achève sur les difficultés des survivants descos erson le départ des Juifs des pays arebes en dumonde. d’Irakin Juifs à quiter un las ov Le livre est divisé de manière chepus 2500 ans.

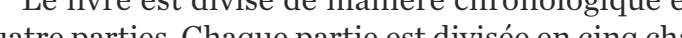
quatre parties. Chaque partie est divisée en cinq chapitres autour des mênes hines. les faits appuyés sur des dates, les homnes, les lieux, les papiers (passeports, visas, courrier, liste de rescapes), les problemes. Les cartes etsurtoutles nombreuses photographies histoliées au texte, très soigneusement légendées. Bref il s'esit d'un livre destiné à toute personne de bef, sogit dé l'importance de l'appareil de notes. C'est un livre clair dont la lecture facile est particulièrement utile pou ercevoir, avec la tragédie de la déportation, un aspect de l'immense quantité de souffrance provoquée par la politique des nazis et l'indifférence ou l'impuissance les témoins dans le monde.

Jean-François Forges

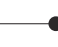

\section{SOLDATS, COMBATTRE, TUER, MOURIR : PROCÉS-VERBAUX DE RÉCITS DE SOLDATS} ALLEMANDS

Sünke Neitzel, Harald Welzer

Gallimard, « NRF Essais », 2013, 640 p.

a parution en Allemagne en 2011 de ce livre coécrit par l'historien Neitzel et le psychosociologue Welzer a fait sensation. Il vient dêtre traduit pa s'intéresse à l'histoire de la Seconde Guerre si lo míntere à histoire de la Seconde Guerre mondiale (période qui n'est précisée ni par ) et de la Shoah.

Les conclusions que tirent les auteurs de leur étude peuvent être critiquées. Au départ, il y a la « chance du chercheur qui n'est jamais due tout à fait au seu hasard. En 2001, le professeur Neitzel tombe dans les claves anglaises, sur des comptes rendus découtes danc dats et hauts gradés des trois armes - dans des camps en Grande-Bretagne. Cet espionnage de prisonniers, trinement eu lieu à toutes les époques, mais à certe tainement eu lieu à tón à les époques, mais à cette occasion il fut pratique a une échelle " industrielle ». fus littéralement aspiré dans l'univers de lasion: « Je fus lín se déployait devant moi.» [p. 10] Léchantillon est trè ample, il sagit de conversations de milliers de prisonPar la suite, Neitzel découvrit dans les archives Périr la suite, Neitzel decouvrit dans les archives de la même façon, deux fois plus important coctés de la même façon, deux fois plus important. Au total ce furent donc près de cent cinquante mille pages

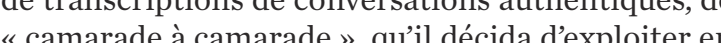


collaboration avec son collègue de l'Université de Giessen, Harald Welzer qui jouissait déjà d'une grande otoriete pour ses travaux antérieurs. On peut consiperrer, avec les antenrs, que les prisonniers n'etaient pascon ix cent. Le res six cents pages. Les auteurs procédent dabord a une lly port II $^{\mathrm{e}}$ Rei (chapion plus an plus long, aborde en une cinquantaine de rubriques tour qui la gue de con fie et qui se lassent pour qui la guerse est finie et qui se laissent aller : ils on' est la guerre, par exemple celui de pouvoi suprême quest la guerre, par exenplecel de pouvoir nitrailler des civils en fuite quand on est aviateur, de coule bateau quand on est sous-maninis

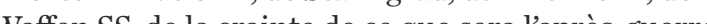
Waffen SS, de la crainte de ce que sera laprès-guerre. La conclusion de louvrage contient quant à elle des . fascination. L'horreur est docume touche d'authenticité.

Les exécuteurs, les bourreaux, parlent à leur alter ego sans aucune retenue et on peut le supposer, sans dissimulation, comme ce caporal qui a exterBelsique en 1940. Ils n'expriment évidemment pas oind en moindre regret. Les soldats qui sont allés sur le front par eux-mêmes ou dont ils ont été les témoins. Sur « parternlextermination » (les auteurs, pour des raisons peu cón con úm de ces « tenoins oculaires» est presque insoutenable. ins la var scies timable: on apprend par exemple que les exécuteurs touchaient une prime. Comme lecrivent les auteurs in système d'extermination. $[\mathrm{p} .210]$ Lapre rieur du système dextermination. » [p. 210] L'apport decisitutive pour la recherche est de prouver, par déclarations prononées devant des enqûteus ou un tribuns prononcées devant des enquêteurs ou tex mands savaient que les Juifs étaient exterminés dan Timothy Suder) Reppelons que dix-sept million de Tho hy Snyder). Rappelons que dix-sept milhions de 1945. Citons les auteurs :
Pour résumer, on peut dire que l'extermination des Juifs etunélément de l'univers cognitif des soldats, et ce, dans une bien plus grande mesure que ne permettraient de lattendre s études récentes sur le sujet. Il ne fait aucun doute que ous ne savaient pas tout, mais les procès-verbaux découte revelentent tous les détails de l'extermination, jusqưaux mises à ort par noxyde decar on foule de to ro ation, raison pour lacullo onpent aussiconsidere, dansce tués. [p. 180]

Par ailleurs, il est remarquable que, sans doute par solidarité de situation, beaucoup de ces prisonniers solidarite de situation, beaucoup de ces prisonniers le livre aux prisonniers de lAs'ilée rouge. Cependant, écer un tès prudo dan la très prudents, mais ils ne résistent pas toujours à la cour souhaiter (tante plation de la violence guerrière ». Sur cette question et sur la remarquens Coquio sur le et fre Coquio s'était lot

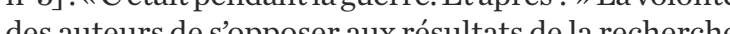
historique la plus récente les historique la plus récente les conduit a proposer de arguments parfois douteux, comme, par exemple, delextermination des Juifs par Himmler levocation delext cours de Posen «qu'elle netait pas le centre menta 190] 190 Ou encore cette fâcheuse tautologie: «la majorite toutefois es téc du terme, Nia), du terme, Nda), mais celle de la guerre. » [p. 501] Et "pour quicon's peut "gas dire qu ils aient mené, de leur point de vue, n [p. 356] Lexpression «deleur point gueverat. [p.356] Lexpression «deleur point de vue» est le pivo de largumentation: a la limite, les soldats de la Wehrmai qui ont participe à des massacres de Juifs n’étaient pas et Welzer insistent beoucoup sur la dinension de guerre comme « travail» pour les soldats, ce qui contribue fortement à cette " désidéologisation ». Au fond e repro est d'avoir privé cié l'approche socio psychologique est davoir privilegie lapproche socio psychologique au détriment de lapproche historique. La définition du u justifier de "partisans » qui a servià ordonner et à «justifier» les massacres sur le front de lEst et en nurope orcidentalé (Oradour, les Fosses Ardéatines) n'est jamais proposée par les auteurs, alors même qu d'êtrelà Lelivre ses victimes qui avaientenle seul tort detrela. Lelivesen, sur ce point, Ignorer le remarquab́c traval de mónóne mené par Hannes Heer et sonequipedans lesant

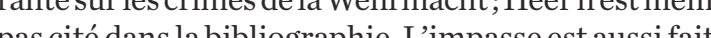
pas cité dans la bibliographie. Limpasse est aussi faite idéolog cos 193-193 etlintensecondionnement des crimue quia faitdebeaucoup soldats allemands des crininels de gurre et des gentrocidaires. En ce qui concerne la traduction, on peut contester le choix de "ethnie » pour traduire Volk, il ausit fallu conserver "peuple », plus en accord aveclicélogie nazie. Mais, encer

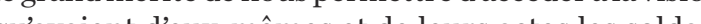
allemands de la Seconde Guerre mondiale. Robert Kahn ignorance cynique du passé. » C'est donc entre deux cueils, entre la sidération devant un passé terrible et la fausse conscience de ce passé refoulé, que créateur et pectateur doivent passer: mais l'art devrait permettre d'échapper au dilemme.

C'estlà précisément que se situe l'ouvrage collectif dirigé par Alain Kleinberger et Philippe Mesnard, qu propose un très riche panorama de ces « représentaton a tourques. Quand conm du d'Europe? Dans quels pays? Avec quelle restion? Qurope Dans quels pays? Avec quelle réception?

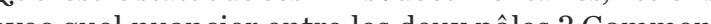
montrer, et que tre leses ? Telles sont quelques-unes des questionsquelouvage permet de poser

es auteurs sont partis du constat que les fiction sur la Shoah se multiplient, surtout au cinema, à un cos premirs filon pourrait croire, n'est pas récent; les de la guerre. Dans cette première génération de films, Ariel Schweitzer atle précé genér (1947) un des premiers films isráliens deyer Levin

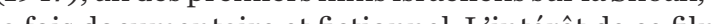
a fois documentaire et fictionnel. L'interêt de ce film (1961) le cinéma israélien tend à ignorocès Eichman (1961), lecinemaisraclientenda igne lui-mên int utilisé des extraits de ce film comme imaes d' ont utilisé des extraits de ce film comme images d'ar-

Au même moment Wanda Jakubows

Aüméne moment, Wanda Jásowska tourne $L a$ Derniere Étape (1947-1948), analysé par Alain Kleinberà. Jirk à Birkenau en 1943. Le film, tourné à Birkenau, avec pour figurantes danciennes déportées, est critiqué par culá que la majoritédes victimesétait juive. Pour Kleinberge tation vient d'ailleurs, car après-guet. Mais loccultation vient d'ailleurs, car après-guerre en Pologne, «les victimes étaient, rétrospectivement [...] considerees comme polonaises. $\gg$ Message également quelque pe Frank Stern: Staudte (1946) pose bien la question de la culpobilite Staudte (1946) pose bien la question de la culpabilite allemande, mais « coupable de quoi, au juste? » Néanmoins, quelques-uns des meilleurs films representan nés à frach 'égitime, et jusqu’à quel point? Omer Bartov semble admirablement pointer le dilemme, quand, a propos

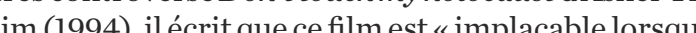
exp à a fois les efets destructeus de la expose a la fois les effets destructeurs de la mémoire et de la conscience contemporaines causée par une 
d'expérience cinématographique, de technologie, de concentration artistique et de mauvaise conscience. ' Du côté français, Antoine de Baecque interroge God a la Shoa. Godard refuse la representa Gothétiqu la she le mo re esthétique, cherche le montage juste " qui permet de representer charchear lexternination. Ses choixmilipest palestiniens en 1969-1 sur les pouvoirs de lart: Histoire (s) du cinéma stig. la monté des perils, et lui-mêne se juge coupable:

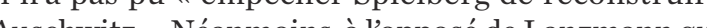
che l'archive l'image d'époque (inexistante ou refuse larchive, limage dépoque (inexistante ou truquée), Godard insiste sur la présence des images de ition bies sonch le la tragediedusiecle. L'oppode Notremusique nest loncpas un de Notre musique n'est doncpas uniquenent politique, ou, pour mienx dire, ellétiques. Ce qui est dévelion politique des choix esthétiques. Ce qui est développé a a la rorly (qui do rôle de la résistance politique à Auschwitz) et de Rémy Besson qui synthétise le genre de Shoah en repren

Cette question traverse, peu ou prou, tous le articles, puisqu'il sagit, comme le formule JeanMichel Frodon, de «La Shoah conne discriminant

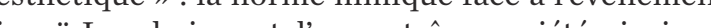
inoui. Les choix sont dune extrême variété, ainsi que es interno distinger quelques lignes de for

Ener quelques lignes de force.

En prenier lieu, le documentaire est-il un « document » neutre ? Luba Jurgenson démonte, ave lexemple de Theresienstadt, les mecanismes de propur commande des nazis. Philippe Mesnard explore sur commande des nazis. Philippe Mesnard explore la «zone grise » (Primo Levi) des Sonderkommandos, our ces de la lutte armée, aux résistapposition aux héros de la lutte armée, aux résistants. Annette ou Jan Karski de Yannick Haenel, et la o tion tion» du personnage historique: «L héroíque courrie catholique de la résistance polonaise s'est transforme catastrophejuive, en Justepariles nations, et en quasi-Juif. » Il ressort de ces trois articles que le documentaire n'est pas plus « objectif » qu’une fiction nais procède aussi de choix esthétiques et politiques. En second lieu, ce qu’on pourrait appeler «les défornations nationales . Pour INllemagne, Matthias Steinle montre comment, dans un processus d'autovictinisation, les docudrames allemands deplacent en flanpos dis d'une fentre (Le Pianistante en flammes qui saute dune fenêtre (Le Pianiste de une équi cstransposédans Dresden (2006), suggérant une équivalence entre le bombardement de Dresde et le ghocide, ou encore, les corps enpiles du Lager

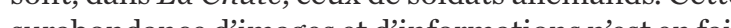
surabondance dinngest en fai que le symptôme et le moyen d'une nouvelle phase de Poment du passé.

Pour l'Italie, Millicent Marcus rappelle que, dans la mesure ou $80 \%$ des Juifs italiens furent sauvés l'Italie evite de se confronter au rôle qu'elle joua dans lHolocauste, alors que les lois raciales de 1938 sont Ur initiative de Or lecinénaitalien, pourtant tres engagé sur des sujets sociaux, a tardé á s'enparer du sujet. Lauteur suggère une interprétation incess sion de URSS the lissolution du PCl amènerait une nouvelle façon de penser le phénomène fasciste a parti de la perspective juive et, dautre part, lantux dimmigrants du Tiers monde obligerait à poser la question la tolérance à l’autre.

Pour la France, comme pour l'Italie, le sujet a été longtemps occulté. (Il faut mettre à part Nuit et brouillard, auquel plusieurs auteurs font allusion, mais qui reste un phénomène isolé à sa date.) Ce n'est que dans les années 1970, après Le Chagrin et la pitie (1971), que le cinéma de fiction commence à s'y attacher Anny Dayan-Rosenmann inscrit Monsieur Klein (film français de Joseph Losey), dans la lignee des films de annés 1970 quicommencent en France, conme Les Guichets du Louvre ou Lacombe Lucien. Lauteur soulignelaveuglement du protagoniste, qui ne « voit » rien alors que le film nous montre les préparatifs de la rafle du Vel d'Hiv. Pour la Hongrie, du románonyme d'Imre Kertészans destin, adapté du roman éponyme d'Imre Kertész avec la collaboration delauteur, et dont la réception négative contraste avec le succès du roman; elle suggère de replacer le film dans lhistoire du genre, et voit un « symptôme pays. En transversalité avec différents pays justement, le passionnant article d'Omer Bartov, «Le "Juif" au cinéma: du Golem à Don't touch my holocaust », étudie dese

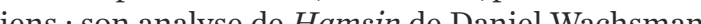
(Isräl, 1982) me paradoxal, des stéréotypes sur le Juif et l'Arabe.

En troisième lieu, les stylisations artistiques. Eexemple analysé par Magali Chiappone-Lucchesi $(L e$ Vicaire de Rolf Hochhuth et son adaptation filmique, Amen de Costa-Gavras) reste globalement proche du réalisme, même si le texte théâtral travaille sur les limites du genre : insertions de documents, longue dides du genter

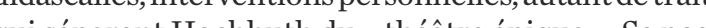
qui séparent 1963 nest la question du public: les spectateurs a forger un à forger une inage, une conscience En revanche, les

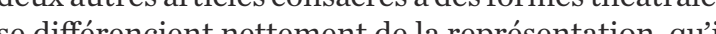
se differencient nettement de la représentation, qu' slisse du travall de Sarah Kane sur le traunatisme (Elsabeth Angel Perez) on du (theatre defigies" de Meister) qui, par la manipulation de figun hes dans une mécan du camp, anòne les acteurs à entrer dans e

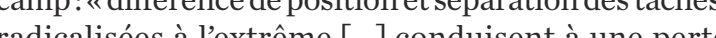
ra totale du sens moral des actions. " En rester la serait evisenit comme le formule Walter Benjameurs, mais il s'agit, comme le formule Walter Benjamin, d'arracher limage au continuum de lhistoire universelle, seule condition dun regard critique. " faut jouer à » et non pas sidentifie

Dans tous les articles, se pose, implicitement ou non, la question de la réception, laquelle renvoie son tour à celle des intentions de lauteur. Christian Bret, devant la pièce Anathène, de la compagnie belge Groupov (Avignon, 2005), voit Auschwitz, cela contre de même, lisant le roman de Philip Roth Nemesis, ily de même, lisant le roman de Philp Roth Nemesis, il y voitune allegorie du camp, commeletait La Peste. Dan es deux cas, sa uvision » est convaincante, et rappelle que lintention de lauteur - longtemps abandonné par cost qu'un paramètre parmi d'autres de la retor - nest qu un paramè jo pami dautres de la signifcation dune œunre. Âr pinverse, il peut arriver que

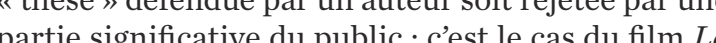
Question humaine de Nicolas Klotz (2007), analysé par Stéphane Bou. Ce film, adaptation du roman éponyme de François Emmanuel, s'inspire notamment de Modernite et holocauste de Zygmunt Bauman, qui étabit formules-chocs, comme "Personne n'ose dire que

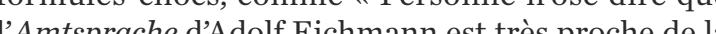
lamtsprache dAdof Eichmann est tress proche de la de l'or neve chente

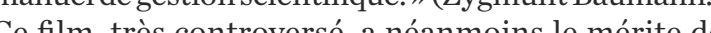
Ce rappeler le rôle du grand capital dans la montée du ?anch Lor

La controverse a également fait rage autour de $L a$ Vie est bellede Benigni.une partie du publicn'acceptait pas cette vision ludique et onirique du camp. En marge Susan Rubin Sur Inglourious Basterds de Tarantino, Susan Rubin Suleiman s'interesse au film de Benigni, comme à Train de vie de Minaileanu : ce type de film toire telle quelle aurait pu être, et quico cu de lhistoire telle qu'elle aurait pu être, et qui coexiste avec la connaissance tragique de ce quelle a été. »Ces repréentions cont pas de nature à encourager le négationnisme: a histoire, c'un ciné que put d'istore qu'un cinéaste peut se permettre « des écarts réel tout belle réflexion nous incite à envisager l'histoire et politiqu Anne Roche 
comme le fit déjà Tzvetan Todorov (cf. Les Abus de la mémoire, Paris, Arleà, 1995) - sur les différentes expressions des dérives de la mémoire.

Après une brève introduction sur le rapport entre la mémoire et 'histoire- rapport qui n’a pas toujours été compris et interprète correctement dans les célébrations officielles - lauteure choisit de s'attarder sur trois le discours public : la négation, la la mémoire dans te discours public: la négation, la sacralisation et la tis tincts dont ellemontre tout for les connections: «Ces trois abus s'enboitent connme les pièces d'un puzzle, (c) es protagonistes rendent compte.

En résulte une mémoire collective (qui ne concern pas seulement l'Ttalie) «exposee aux feux croisés » de diverses, dont laction suscite, en général, detivations diverses, dont laction suscite, en général, des controverses, des conflits ou des déformations de l'histoire (sans parter déla mémoire). Et c'est aussilaraison pour en rellan en renonçant à approfondir (du moins dans le débat pub́c et lans lusage courant) le rapport entre notre

présent et notre histoire, nationale et européenne.

La reconstrútion de ceśris formes majeures de dérives de la mémoire se présente conne une reconaissance synthetisce des dinesrestes étapes qui ont

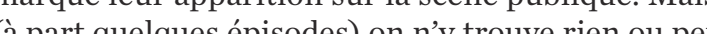
(a part quelques épisodes) on n'y trouve rien ou peu sur le débat critique que ces étapes ont provoque, pa exemple chez Primo Levi (pourtant cite), ou sur les extes histor ituries, ences ont accompagné une réfexion critique sur les ouvrage des auteurs du négationnisme, de la sacralisation et de da banalisation. On ny trouve de même aucune trace ont généré par opposition des nouvelles études et de . nouveaux acquis, par exemple en matiere de fonctionlogiqu "sur les sites du génocide. Ens

En conclusion, louvre de Valentina Pisanty est un bon outil de vulgarisation. Mais, contrairement à certains de ses autres ouvrages, le texte noffre pas deléments donton

\section{MEIN KAMPF, \\ HISTOIRE D'UNN LIVRE}

Antoine Vitkine

Paris, Flammarion, «Champs histoire », 2013, 332 p.

originalité de l'étude d'Antoine Vitkine est de s'intéresser au livre Mein Kampf en tant que tel, cest-a-dire a l'histoire de sa publication, et de sa cepion,

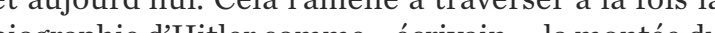
pographie dittler comme «écrivain », la montee du hazisu la la sours sous 'angle des effets de ce livre qui n'est pas comme les es articulier l'antisemitisme) que par son impact.

'auteur étudie d'abord les ventes du livre, importantes dès avant la prise de pouvoir, et son accueil en 列 mépris mépris. Aucun groupe, aucun parti ne semble prendre niste iste, les Litles, oumênelapressejuive. Mais en 1933 quand Hitler devient chancelier, un million d'Allemands achètent Mein Kampf, avant même que l'Etat lo lance une canpagne de propagande. Les mairie loffrent auxjeunes mariés; le partinational-socialiste mais a a ou l'offrent. L'endoctrinement de lajeunesse étant une priorité des nazis, on publie des livres d'images qui en Aretranger, a

Al'étranger, après 1933, le livre est traduit, mais dan des versions expurgées de ce qui touche à la politique étrangère. « Sij’avais pu deviner en 1924 qu’un jour je deviendrais chancelier, jamais je n’aurais écrit Mein Kampf » aurait confié Hitler à un proche: le texte dévoile le gêner du moins tant que le rapport de forces neu le gêner, du moins tant que le rapport de forces n'est pas en faveur de lAllemagne. Il veut donc restreindre laccès des pays étrangers a son texte. Ceux-cis'alignent parfois sur les desis de lédteur allemand: c'est ains quaux USA et en Grande-Bretagne, les éditeurs publien En une version censuree par Eher-Verlag.

En 1933, l'Italie mussolinienne achète fort cher étant juif,Bompiani fait disparaître son nom. Les moti- vations de l'éditeur sont obscures: s'agit-il d'avertir les Italiens d'un danger, ou de les endoctriner? La même ambiguïté se retrouvera en France.

En effet, la France est particulièrement attaqué dans Mein Kampf.Or Hitlerveut rassurer l'opinion frança . de Brine (for firme pacifiste et minimise Mein Kampf, « un livre plein dimprécations écrites en prison. » Et il refuse looff́ e tradion de tionslatines. Celui-ciestun curieux personnage: attire par le ascisme, lia adhere aum minemente

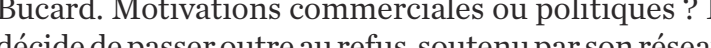
décide de passer outre a refus, sontenu par son résea au ministere dXnciens Combattants. Au passage, Vitde Lyautey « Tout Français doit lire souvent cité éc ryantey, "Tout Français doit lire ce tive ", qui a après le procis, la Confédération des co tribuables,

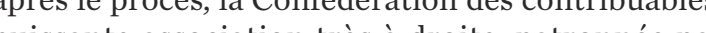
puissante association très à droite, patronnée par interdit aux Fro un chan a chancelier an pros de deux tiers nde ses idées, en particulier franc-maçonnerie, la presse. Et Lyauteyles en felicite... ce qui donne à sa phrase une certaine ambiguité, et $p$

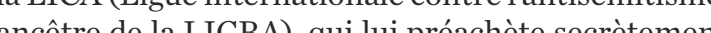
cinq mille la LICR), qui lui préachète secrètement cinq mille exemplaires. Ces exemplaires sont envoyés à des parlén Calares, l'un dres, avocats, etc. Áce propos, Andre Calmettes, lun des traducteurs, témoigne: Nos députés n'ont pas le temps de lire los exmplir. cos députés n'ont pas le tenps de lireles exenplaires que nous retrouvons en grand nombre chez les bou-

quinistes sur les quais.» [Témoignage amilial, inédit] Hitler porte plainte, la Société des gens de lettres sassocie a sa plainte. Lors du procès, en juin 1934 lavocat de Sorlot souligne lintéret de faire connaits Frane Le tribunl ne le suit pas. Mais l' haine de très relayé dunal ne le suit pas. Mais linterdiction, très relayée dans la presse, accroit lintèrét pour le livre. Sorlot publie une brochure dextraits, portant un bandeau "Le livre interdit aux Français » et contreattaque « Les droits dauteur de Hitler? Mais qu sest donné le ridicule de songer aux droits d'auteur brûlé publiquemş lont on a interdit la lecture brúlé publiquement les ouvrages ? " L'argument est suite le prouvera. Il éditera désormais des ouvrages favorables à Pétain et à la collaboration, mais continuera à vendre Mein Kampf clandestinement, ce qu lui servira à la Libération lors de son procès : d'Astie de la Vigerie témoignera qưil « a fait son devoir de trançis en servant à éclairer la Résistance en livrant es exemplaires de la bible nazie.

Comment les Français reçoivent-ils cette lecture, intégrale ou en extraits ? On est frappé, à près de uatre-vingts ans de distance, de l'inadéquation des eactions. Les politiques pensent qu'il s'agit d'un livre de jeunesse », écrit avant d'être au pouvoir ; l'armé voit un simple remake du projet bismarckien, de la volonté de puissance prussienne. Certains pensen u'Hitler en veut surtout au communisme et à l'URSS, e qui n'est pas pour leur déplaire. L’aveuglement n'est pourtant pas général. Dès décembre 1933, Trotski, qu deHitler et démonte son prétendu pacifisme, dallitich journel Lárité, dontlaudiencest joun Suarès dac $L$ Revue littéraire, mais ils no entend En 1938, peu avant Munich, l'éditeue Hitler, Eher-Verlag, fit pulie Ma Doctrine, chez Fayard Il rerit d'une sorte de « versio Doctrine, chez Mein Kampf rédigée par deux journalistes qui toutes garanties : l'un est à Candide l'autre à Je suis partout! Le texte est toujours antisé late, a Je suis partout Le texte é toujours antisénite, mais efface toute agressivité vis-à-wis de la Trance. Dailleurs, deux mois apres Munich, le 6 décembre, est signé un accor Aveclaguerre l'inténêt pour Mein Kampfs'r

Avec laguerre, linterêtetpour Mein Kampfs' intens que: en nu quarante-cinq ouvrages de resumes, extraits, analyses. Értains sont des mises en garde, dautres, comme les des éloges du “ visionnaire \# Hitler; l'auteurchin, Eles clogen conde à mortà la Liberation.

Après la defaite de lAllemagne, lors des procès de Nuremberg, le livre estutilise comme preuve à charge Mais les Allemands, qui lont presque tous possédé, lanténégation cest dificle à établir, vu limportance de d'Ingeborg Rabenstein-Michel, «Adolf Hitlers Mein Kampf gezeichnete Erinnerungen an einitlers Mein Kampf, gezeichnete Erinnerungen an eine große Zeit : veur par et dón veur par et dans la BD », in Témoigner entre histoire et dé́tudes nes 113. Cequi explique en partie qu il y ait pe part, on l'a vu, il existait de nombreux résumés, sou 
diverses formes, qui ont «imprégné » l'ensemble de la population, même sans une lecture intégrale du livre. Certes, et lauteur le souligne, lidéologie antisémite qui a prepare la destruction des Juifs d Europe ne se trouvait pas uniquement dans Mein Kampf. Mais le la fó

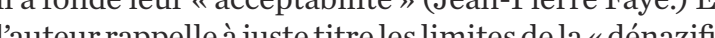
la

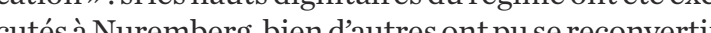
cutés à Nuremberg, bien d'autres ont pu se reconvertir dans le privé ou dans la politique. Ains, le secretaire gènéral de la chancellenie didenauer, Globke, fit

L'auteur exam d'Allemagne, après la guerre. Hitler étant resident bavht du livre pour l'allemagne entiè reç le copyright du livre pour lAllenagne entière et la mission il ildonneraitunema

Dans certains pays, Mein Kampf est interdit, au le Baviere, régulierement faire retirer le liv a de la vente en Russe, a róssi a

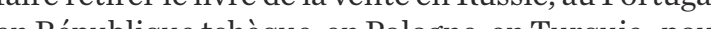
en République tchèque, en Pologne, en Turquie; pour tions ontété publiées ces dernières années le livre

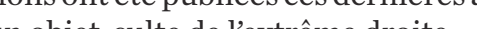
nobjet-culte de lextrême droite.

Dans dautres pays, lachat du livre est légal : en Italie, aux USA, en Angleterre. En France égalemen alors que beaucoup croient encore qu'il est interdit. dorot, réhabilitén cus,publiedestextes de lextrême droite, y compris négationniste (Rassinier) et contipàs 1960 , ouver après 1960, ouvertement. En 1978, dans un contexte de de de Darquier de Pellepoix), la LICRA porte plainte pour faire interdire Mein Kampf, alors quelle avait paye pour diction, mais exige l'encart d'un avertiss pas dintercheque ex chaque exemplare. Fayard prepare une édition criique pour 2016

En Allemagne, le livre reste interdit, sauf aux chercheurs et historiens accrédités; seule est autorisee publication dextraits connentés, dans un but scienpublic. Li situthis

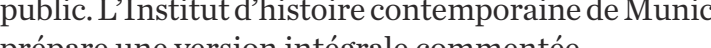

Hors d'Europe, «dans des pays où le nationalisme s'exprime sans la retenue que donne l'expérience de l'Histoire » (comme le dit gentiment l'auteur), Mein Kampf a un succès populaire : en Inde, en Asie, en Afrique francophone, dans le monde arabe. Lauteur rappelle que dans les annees trente, le nationalisme terre, la France Sion lan un best-seller, loin devant Mein Kagesde Sion sont un best-seller, loin devant Mein Kampf, ce dernier se vend toujours dans le monde arabo-musulman, surtout sous forme enquête de terran faite par lauteur Turquienon delecteurs lont reellement lu, ils syreferent, de façon (u) Anfit israelo-palestinien.

Antoine Vitkine a défini son travail, dans une interview, en termes modestes : « Ce n'est pas un livre dhistorien a proprement parler, plutôt un livre de jourdit dit, sur des sources allemandes et françaises, au preminer Buches : Mein Kampf 1922-1945 (Oldhich einese Buches : Mein Kampf 1922-1945 (Oldenbur. 2006), la thèse de Josselin Bordat. Mais son ouvrage a le mérite de mettre ces informations a la disposition du public, de façon claire et argumenté. Et il prend parti dans le déba dire Mein Kampf mais le décoder, compras interdire Mein Kampf, mais le décoder, comprendre le conditions historiques de son apparition. Et en même temps, savoir que histoire ne se répète pas, ne pas faire ces anhos tronte un cétre capable deconprendre lanouveautédunévénement. ne peut quêetre d'accord avec ce programme. I

$$
\text { Anne Roche }
$$

\section{HITLER ET LES PROFESSEURS}

Max Weinreich, Samuel D. Kassow, Martin Gilbert (Préface), Isabelle Rozenbaumas (Trad.) Paris, Belles Lettres, 2013, $393 \mathrm{p}$. ax Weinreich ? À scruter les index biblioaphiques d'ouvrages qui ont connu (et tomes de La Destruction des Juifs d'Europe de Raul Hil- berg, ou qui ont nourri la controverse, tels l'ouvrage de Daniel Jonah Goldhagen, Les Bourreaux volontaires de Hitler, et, plus récemment, le livre de Christian Ingrao, Croire et détruire - dont le sous-titre est Les intellectuels dans la machine de guerre $S S-$, la tentation est forte de répondre : personne ou presque, tant brille par son absence le nom de Max Weinreich. Pourtant quand Raul Hilberg commence en 1948 ses travaux de état-Unis, sa some le livie de Max Wein anx Éta est paru depuis deux ans à New York, en yiddish Hitlers profesorn - et en anglais - Hitler's Professors même du yiddish en anglais. Aussi la publication de ene du yivis en

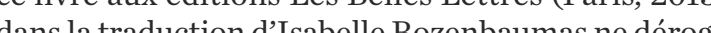
dans la traduction disabelle Rozenbaunas ne déroge pas au caractere savant sinonéndit qui est la marque cet sich vall de bien des rection du national-socialisme, on peut penser, a priori, mais carsent rescapés ne sont pas restés « les bras croisés ».

Les quelques noms familiers en France d'intellecues quelques noms familiers en France docteurs d'Université compromis avec une tués ou de docteurs d Universitécompromis avecune idéologie en train de conquérir les esprits et les masses - Junger, Carl Schnitt, Heidegger- disparaissentensevelis sous láćs gen gravoir ils mières. Zésés gens de savon, lls sactivent, dès les premondiale a thèses rampantes du nazisme. Weinreich mone thè ses rampantes du nazisne. Weinreich montre

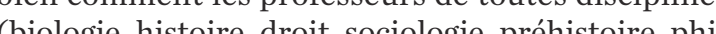
(biologie, histoire, droit, sociologie, prehistoire, philosophie) font sans hésitation le décisif premier pas enction rière ou affinité idéologique, ou les deux réunis, cariere ou à ne compte guère. Compte ce qui en rèsulte : à partir de 1933 '’ stupéfiante proliferation dinstituts, de centre deetudes et de documentations, de fondations à développer l'étude de la question juive, à fes races. à développer létude à la question juive, à fabriquer une science anti-juive, à étudier l'influence juive su "la vie eccléslastique en Allemagne ». On n'en finit pas de recenser ces lieux, ces tribunes où se prépar de race, d'espace, d'espace vital, de sang, de Juif et qui vont nourrir le programme de conquêtes des territoire à l'Est du Reich et les effroyables menées exterminatrices qui les accompagnent

Max Weinreich ne problématise pas, au sens où on 'entend aujourd'hui, la documentation qu' il a rassemes faits » est son mot d'ordre. Les faits ce sont bie toriales, les massocres. Sontégalement de l'ordre des its lives ins 列 le jor de l'authenticín sommedisait le jargon delauthenticitè conme disait Adorno. Ily Rosenberg Himmler, ettellement d'a in itutscol ensuri d'or dorganismesproducteurs de rapports; jamais assez de débusquer, traquer à l'insu même du plus aryen des commes, hommes, ce qui est juif. Il y a les textes, les faits et par le Fran fort en : 1941 les congres jusquen 1944 de Trancsort en iars 1941, les congrès jusquen 1944 Cracouting Cracovién

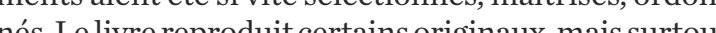
Wein. Livich reproduit certains originaux, mais surtout

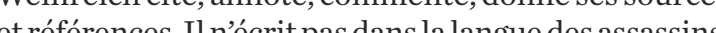
mis dan celle des victimes langue parlée lue, danses

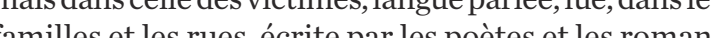
familles et les rues, ecrite par les poètes et les romanViers.leyiddish. Langue particulièrement al honneur Vilna (Vilnius, Lituanie) avant linvasion nazie, langue célébon tour la traductrice, Isabelle Rozenbaunas, clestre da Ceuple pour un peuple sans langue.

Cest cela que dit en presque dernière phrase de son livre, au bout d'un travail mû par la colère et la protes-
tation furieuse, l'arpenteur Max Weinreich :

À supposer que lon plaçâtles individus assassinés en ordre de marche, la file de squelettes s’étendrait de New York à San Francisco, puis encore une fois de San Francisco jamas ces victimes de l'Ordre nousqua Chizo. Et Jamaiscesvictimes de «IOrdre nouveau » pouvaient seusementeten un regard sur 1 Europe de lapress-guerre, elles quiétions, paraît-il la cause de tous les mare ironie : nou plus là, porat-il, la sausedetous les naux ne sommes plus là, pourquoi n'y a-t-il toujours pas de quiétude? 


\section{NOUVELLES PERSPECTIVES \\ SUR LA SHOAH}

van Jablonka, Annette Wieviorka

Paris, Presses universiteres de France,

La Vie des Idées », 2013, 128 p.

e champ d'études qu'est la Shoah est abordé et traité de différentes manières du fait d'une multi- tude de sources, de questionnements et de formes verce dinis le C'estete faniliale. Les possibilités sont nombreuses. est justement lobjet de cet ouvage coordonné par '

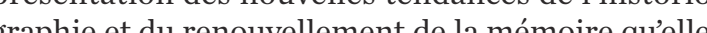
raphie et d mpliquent.

Annette Wieviorka installe le cadre de ces nouvelle cherches en énumérant trois verbes très évocateur pour la mémoire : comprendre, témoigner et écrire. lifier l'histoire juive, tristement célèbre, elle sour quale véritable boom de la mémoir de la Sho soulign le véritable boom de la mémoire de la Shoah »[p. 6] écir . pionniers des recherches, à savoir Raul Hilberg et món migrent selon les generations dhistoriens. Si les prearchives en 1997 a multiplié les études et leur pe

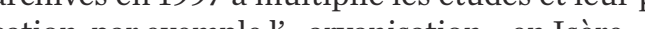
cation, par exenple l" aryanisation » en Isère.

Annette Wieviorka insiste aussi sur le fait que les hictimes et rescapes de la Shoah sont les premiers historiens, puisque ce sont eux qui ont entrepris des

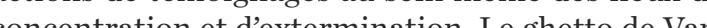
concentration et dexternination. Le ghetto de Varsovie en est un exemple. Une étude est cependant nziet

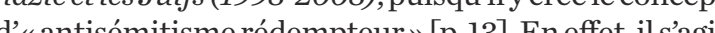
d'uantisénifismécréch une histoirécrite de tous les points devue: celui des Allemands, d Hitler aux Allemands ordinaires, celu es soctes dins lesques . Cest la nise en parallele de deux historiographies.
Toutes les études sont désormais possibles pour es nouveaux historiens puisqu'ils bénéficient des trales nouveaux historiens puisqu'ils bénéficient des travaux de leurs annes défricheurs, d'une large ouverture darchives te la possibilite daller consulter temoin et archives a lautre bout du monde. De ce fait, on trouve de plus en plus de quêtes familiales sur la vie Jentes peu ou pas connus.

intéressé aux archive nazies en Allemagne, dans la ville de Bad Arolsen. C'est là que se trouve le Service international de recherche (SIR). Cette institution, creeee en 1948, devait termiet de ré de la de la guerre. Lobjectif premier de ce service etait une priorite donée aux recherches des familles et non aux de l'inces. Existantencore aujourd hui, «elle témoigne de linessante quête de mémorre, du travail de deuil jánais interompu, mais aussi des procédures de réparations allemandes. » [p. 19] Les activités du SIR sont nombreuses. recensenent des tombes et fosse tion d'archivesomagenents des victimes, classificationd a archives (on ne comptabilse pas moins de 17 millions de documents : du SIR sont ouvertes à la recherche depuis 2008 . Si les documents nouvriront probablepermettro de nouveaux chanps dinvestigation, ils perinttront cependant de completer les recherche existantesetserontunesource

Tal Bruttmann s'est penché sur la Shoah dans les bureaux et, plus précisément, l'application de la politique antisémite des administrations sous Vichy. Il sagit discrimination : instructions, correspond an discrimination. instruetions, correspondances entre services ainsi que des formulaires. «Chaque adminisà intégrer l'a tisémitismes [p.41] En été amenée à intégrer l'antisémitisme. » [p. 41] En effet, aucun de son activité professionnelle durant l'Oessé de récit

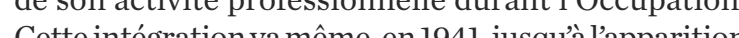
Cette intégration va même, en 1941,jusquả l'apparition jur un formuie de la question : « L'intéressé est-il juires étu est par laires. Les études concernent icitous les échelons de les Juif sont devenus le principl instridentifiant les Juifs sont devenus le principal instrument de leu arestation. " Ressource de lhistorien et enjeux de la mémoire juive, linstrument bureaucratique apparaît

La méthode quantitative pour la communauté juive de Lens est le sujet traité par Ivan Jablonka. Troi moments ont permis d'identifier et de quantifier les Juifs: le recensement de la fin 1940 (par auto-déclaration), l'aryanisation des biens et la rafle du 11 septembre 1942. C'est par ce biais que le processus d'extermination commence et la logique qui la suit aussi, à savo « Un Juif est un Juif, et le sentiment patriotique et l'intégration n'y changent rien. » [p. 56]

L'étude d'Audrey Kichelewski porte sur un ouvrage traitant des relations polono-juives, dans lesquelles les premiers auraient participé aux dans lesquelles les premiers auraient participé aux crimes des second avec comme motivation principale lappât du gain. Capproprato Chettoisation jusquá la denière dent Ce sont «les moissons macabres» [p. 63]. Pour Emajuel pour rendre passible ce type de comportem juifs pour rendre possible ce type de comportement cha-violent à leur ègard. Cependant, la causalite économique de ces actes est relative puisque les Allemanis sétaient, au préalable, déjà servis. Daprès des archives, cest la campagne moyenne qui a pris part a toriogrape toriographie de la Polognecon ces actes, on utilise le terme de "chasse aux Juifs

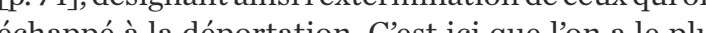
échappé à la déportation. Cest ici que lon a le plus besoin du concours de la population locale polonaise. Enfin, un táci de a ce jour. Kassourdissant silence de llégise pols a ce qui se passait.

Pour Florence Heymann, les ghettos auraient peuttre été des lieux de résistance juive. Pour autant, ghetto representait une étape préparatoire à la Solution finale. Aussi la vie qui règne à l'intérieur de ces juifs, au degré d’auto-admin au juirs, au degre dauto-administration, àla solidarite (ou Pour Moshé Kanovich, les Juifs enferm ́c durvie. ghettos ne ghettos ne se seraient pas soulevés, car «ils savaient qu'ils ne pourraient pas survivre longtemps dans les Ivan Jabond Ivan Jablonka conclut cet ouvrage en evoquan Ainsi, la roure Ainsi, la recherche historique empêche la mémoire de senfermer sur elle-même et de se racornir. De plus, e ces temps de relativisme moral, «1Holocauste sert boussole . Cest un absolu, un absolu du mal. » [p. 93] l'obnubilation, l'anti-intellectualisme, la banalisation, a fermeture et la bonne conscience morale. Cela soulignerait ainsi le caractère artificiel et moralisateur du devoir de mémoire, soit la transformation du souveni n obligation. Pourtant, le renouvellement de l'historographie nourrit une autre manière de se souveni e seul devoir qui s'impose à tous est celui de la vérité. Cest pourquoi un tout nouveau travail est porté sur mémoire, et celui-ci provient des « petits-enfants [p. 102].

Les réflexions menées par ces historiens, à parti d'études d'ouvrages, permettent ainsi de constater le caractère inépuisable des sources et, probablement des investigations que l'on peut mener à partir d'elles. C'est ce qui mènerait ainsi à de nouvelles génération d'historiens qui actualiseraient perpétuellement de nouvelles mémoires.

Anthony Miche

\section{MEMORYLANDS. HERITAGE AND IDENTITY IN EUROPE TODAY}

Sharon MacDonald

London: Routledge, 2013, 293 p.

$66 \quad$ emory has become a major preoccupation - in Europe and beyond - in the twentieth century and into the twenty-first. [...] Europe hasbeconsed with the tion" (1) it is with the ins interyl and its preserva(1): it is withese insightul words that cultura anthropologist Sharon MacDonald opens her latest book Memorylands. The book is an original investiand memory in contemporary Europe. In it MacDont provides the reader with numerous examples of how provides the reader with numerous examples of how texopean identity and memory are constructed, contextralizing all this in a vast and interdisciplinary

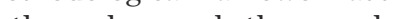
The fophy.

The first half of the book concentrates on how the European past is nowadays made and narrated, felt and then sold. Having introduced the subject of her 
plex, MacDonald first illustrates how anthropologists iscussed the European past in their scholarly work dist int to the a isplayed on Euro curse thropos in the study of Europe disisto antopolin un topics a different as collective id in the rued topics as diferent as collective identity in the rura Argonne region of France or post-Yugoslav Bosnian a are at the ory ory, especially objects, food and homes. fromgardening as a way of re-rooting memory among Greek Cypriot per the mery porary Germany. As MacDonald notes, the memory phen to produc lo to of produced for tourists. This is the case of the heritage entro in 1993 or of the Slow Food move Scottish Is in Ita in 1996 and conlow For in Italy in 1996 and concerned with the pre

In the authenitic food products.

In the second half of the book, MacDonald anayzes specific dimensions of the memory phenomenon: coseum cosmopolitanism and lastly the future of memory Europe. Mus ane a European memorial landscape and, according to the

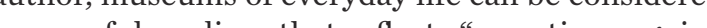
a powerful medium that reflects "an active ongoing the "the museatization of

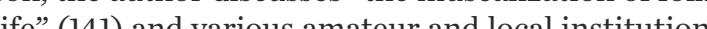
" (141) and various anateur and local institutions such as the Latvian Elhnographic Open-Air Museum

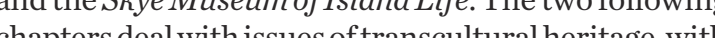
chaptens doal wist bring ebout in bring about in European identity, and then Holocaust memery into when a statue representing a nude of a veiled woman was publicly displayed in the park of the Kunsthalle Nationale de l'Histire de l'Immigra tion of Pas, so to Natin de lHistorede lingmigration of Paris, so to late new and more inclusive ideas of Europeanness. Holocaust commemorations are then interpreted as part of the larger morations are then interpreted as port citizens alike, that for European and non-European citizens allke, that might contribute to reconfigured ways of being national. The future of memory in understers cers

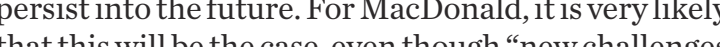
will will no doubtemerge in Europe's menorylands" (235) Cure co to confirm this suggestion, highlighting the need for ret multivocal idea

Even though Memorylands is largely based on articles originally published as separate papers, the autho managed to assemble everything in a well-readable nd coherent way. Inevitably in a book onsuch a broad subject, and as the author herself acknowledges in the . MacDonald conducted fieldwork - feature where macDonald conducted fieldwork nently, as opposed for example to Italy or Spain to which of the memorial lo rad a more detalled discussion with resecially memoration of the Algerian War, which ciena profound impen a profound impact on ideas of European identity and thavare insteadont have also allowed MacDonald to better clarify how the memory phenomenon is not entirely unique to Europe and locations su

his said, Memorylands is a timely and useful tex porary European memory phenomenon, while also in order to much of the scholarly literature needed in order to undertand it. It will constitute essential reading for students and. scholars interested in memhistory.

Dario Miccoli

\section{STORY OF A DEATH \\ FORETOLD: THE COUP \\ AGAINST SALVADOR ALLENDE, SEPTEMBER 11 ${ }^{\mathrm{TH}}, 1973$}

Oscar Guardiola-Rivera

New York: Bloomsbury Press, 2013, 496 p.

$n$ the last few decades many scholars have examine the rise of Chile's President Salvador Allende and the
subsequent coup against the democratically elected socialist. In the context of the Cold War and revolution, Allende's notion of resistance or la vía chilena models. Within the confining definitiona of the Cold War, the Win the War, hie global community viewed Latin Americans as having to choose between U.S. capitalism and Soviet communisn. To many LatinAmericans neither of these alternatives provided truedin Americans a undoubtedly offered Latin Americans a framework to critique U.S. imperialism, the left in Latin America consistently created their own defo the fird a demary a democracy. Aldices and his way of, as GuardiolaRivera clains, "divorcing denocracy and law from

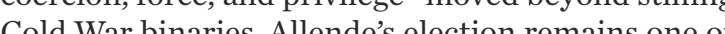
the most significant exmples of homegrown one the most significant examples of homegrown revoluworld view A of change inspired by particular his within C in en by particular historical processes within Chile and the experiences of Salvador Allende. the US. govercent his Popor Unity par a a d w verous challenge US Popular Unity party as a dangerous challenge to m.S. hegenony and capitalism. monograph, Guardiola-Rivera provides one of the to to power, the coup agan this tragic event within Chile.

Guardiola-Rivera's work tells the story of Allend in three parts. The first concerns precedents an causes of Allende's rise to power. Guardiola-River how this in inired him to beconces of Allende and tive for the people" (12). This inspiration came from both theoretical influences and real life interactions with political repression and revolutionary violence. differe experiences inspired Allende to envision a inflive influences reflect the hybrid and creative character of LatinAmerican politics and revolution. In this section, Guardiola-Riveralsolinksticl ences with the greater political climate within Chile. Thise renced within his lifetime and also another layer to what could have been a standard biography. The book is particularly strong when Guardiola-Rivera reference

In part II the Coup, the aut

In part 11, the Coup, the author focuses on the events leading up to Allende's election and the 1970 Rodrítween right wing candiste Jorge Alessand In the beg a r. In the begining of this section Guardiola-River Unity party in the larin Chite a nity party in the larer context of the Cold War. He Mapuche po Alation As president Alles ingenous the peope he would "cspesident, Allende promised the people he would "change the regime and system pla planned to nationalize resources owned by U.S. captop unl stop unemployment, and implement true agrarian War provis promise within the context of the Cold Sar prod nuthe Chilean military officers with the ing a coup. Guardiola ing a coup. Guardiola-Rivera uses CIA documents US. was in subverting Allende's democratic In this sion, which in In this section, which in some ways parallels Gre ica in the Cold War (University of Chice. Lat Ame cain the Cold War (University or Chicago Press, 2004) Guardiola-Rivera explores ideologies about human Guardiola-Pivera Guardiola-Rivera argues that so-called develope rights but only within the narrow context of "c civilized ntions" According to both Grandin and Guardio nations. According to both Grandin and Guardiolaan alther an alternative and a valid path for democracy. The US throw Allende even after a second election reinfored his legitimacy.Ther 
against Allende was a coup against "law and democracy" (238). Indeed, human rights did not matter whe che so-called cillized ally powerful whe world section description of the events leading up to the coup and the

Sectionsequent take over.

Sectionsequences, which is shorter than the other sections, analyzes politica action in Chile up to the present. It examines the rulin Junta and General Augusto Pinochet's "destruction (340). Gular movement, and popular legal politics" "(340). Guardiola-Rivera explores the two types of "and the subsur and the subsequent destruction or human rights. The to ever eve "s in Aatin Anerica dung the Cold War. He - whether soldiers, civil dervants or multination. means the individual liability of human rights viola(35). This powerful statement, hike others in Guardiola-River, work, connects thecoup against Allende to larger his$20^{\text {th }}$ century.

Though the book at times turns into a hagiography of Allende, Guardiola-Rivera's passion for his subject evident. This bookis an excellent choice for anyone who wants togo beyond agenerat Allende bibliography an influences within the complex history of Chile.

Lindsey Churchil

\section{WAITING FOR MARCEL \\ ORPHÜLS: THE LITERATURE \\ OF AUTHORITARIAN RULE AND RESISTANCE IN LATIN AMERICA}

Becoming the Tupamaros: Solidarity and Transnation Revolutionaries in Uruguay and the United States Revolutionaries in

Nashville: Vanderbilt University Press, 2014, 206 p.

The Ideological Origins of the Dirty War: Fascism Populism, and Dictatorship in Twentieth Century Populism

Argentina

Oxford: Oxford University Press, 2014, 216 p.

Memory and Transitional Justice in Argentina and Uruguay: Against Impunity

New York: Palgrave Macmillan, 2013, 319 p.

2010, at the XXIX Latin American Studies Association Congress in Toronto, the historian Steve Stern called 1970s stated-sponsored terror in Argentina a "dirty war". An audience member self-identified as an "Argentine", speaking for "Argentines" scolded him. "Dirty war", he told Stern, was a term invented by the Argentine dictatorship, and as such, should never be uf Austern, perhaps the most authoritative analyst of Augusto Pinochet's Chile, apologized, conceding a truth on that policing language brings us closer to atruth on state terror. The moment evoked the mannertise those who say "Holocaust" for their putative chastise those who say "Holocaust for their putative the Nazis.

As the international scholarship on authoritarian violence in Latin America has grown quickly since 1990, dominant narratives have tended often to rigid binaries on violence, morality, state terror, and even language. As in the use or the term "dirty war", there i investigators racing to fit their cases into these dis- courses - like round pegs into square holes - have disregarded what to an outsider may seem obvious based on the evidence. Commenting on a museum opene in 2007 to remember victims of the 1968 Tlatelolco Massacre in Mexico City, for example, the literary critic José Ramón Ruisánchez Serra writes, "despite a genuine effort to create a site where the most cruevent in the second half of the twentieth centu in Mexico could be commemorated [...] the museun receives few visitors". (Ruisánchez Serra, José Ramón Reading '68: The Tlatelolco Memorial and Gentrifcation in Mexico City', in Ksenija Blibija \& Leigh A. Pan (eds.), Accoun (181) In trying to expla visit this ln trying to explain why Mexicans will not to Ruiśn nez Serra that his starting it never occurs Run hould it the the wong. Could it be that the power acadenic line that Tlatelol was both a uniche and hom state ter mold War Mexicois inacurate? is itpossthe because they do not view Tlatelo

Much of the academic communty addressing Much of the acadenic community addressing authoritarian violence in 1970s and 1980s Latin America has nothad a MarcelOrphuls moment. Some can't see grey zon" os on authoritarian rule - that the tern "dirty war" can have multiple meanings or the che ent Mexicans. This is reminiscent of French politipitié (1969) prompted an opening in how the French pitie (1969) prompted an opening in how the French 1960s, a do 1960s, a doming te Gaullist nave admitted only onemenory those who supported the occupation and there were those whory likely helped Socisist The persistence of that binary likely helped Socialist president François dectes: during the Second World War he had been both a ming dis both a menber of the resistance a in a Vichy bureaucrat, a combination so seemingly incongruous and so at odds with post-war French memory making on the 1964, that wo 1964. As French analysts have come to understand, not only can the Mitterand conundrum not be swept under the carpet. It is precisely that appant contradiction fabing othes
Of the three books reviewed here, two take us long way in breaking down false binaries and dictatorship-era shibboleths (the third less so). Thei mportance rests in the first instance in a rejection of the powerful tendency in the scholarly literature to treat dictatorship as aberrant. They explain violen military rule (and resistance) not as some Frankentein's monster emerging from American Cold Wa nti-Communism but as a function of national an Con long term. Lindsey Churchill's Becoming the Tupamagehammer to obscurantist, false boundmilitary rule and its opponents (in this ase, the leftist guerrillagroup, Tupamaros). This is no heroic opposition to it However, it ogins theoretically and empirically with the hypothesis that to take as a and en the cold wan

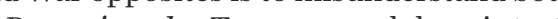

Becoming the Tupamaros delves into the often poorly explained gendered abuse and humiliatio of political prisoners in dictatorship-era Uruguay. Che pexualized viothe against women Tupamaros. She also documents torture methods. But the book also explains bon the violent military and the Tupamaro resist both the violent millitary and the Tupamaro resistance as in Uruguan society. At the same time, Churchill in products of longerm vion in Uruguayan prociety. In their clandestine publications, for ple, the Tupamaros excoriated military authorities for pe, the Tupanaros excoriated nilltary authorities for mprisoning maleguerillas in ageneral prison population that included gay men. The result, according to the f red from a broken window. The Tup a maro was punished with yers of solitery mental illness. In this and other cases, the Uruguay left characterized imprisonment under the military eft characterized imprisonment under the military and "predaty" and "predatory" gay me

Both inside and outside prison, the revolutionary eft in Uruguay and elsewhere in Latin America fre quently shared a prejudiced view of homosexuality Beyond this, Churchill wites, "like the Uruguat. 
military [...] the Tupamaros supported the polarizaion of masculine/feminine and active/passive, wit (51). The and passive as synonymous" (Churchill 2014, 151). The sonetinus of so-called sexual deviance across political divide reflected longer term continuities. Like nonwhitenes homosexuality seemed invisible in post-dictatorship con violence.

Churchill also points out other continuities and connections between post-dictatorship democracy, the tanding overapping political cultures is as important as grasping sharp contrasts. Many have seen the Tupamaros as progressive on the integration of wome into political activity, upen.ing notions of women as ate time, the revolutionary left in Uruguay asked same tine, the revolutionary left in Uruguay asked women conrades to reject motherhood and femininth, and to becons socially constructed nodels of nilitant masculinity. Other leftistgroups stressed women's femininity and maternity or Either way, "enbracing - dininity and maternity or embracing masculinity dichotomous gender restrictions confined women's political participation to const

With the fall of the dictatorship in Uruguay an even more so in Argentina, an initial reconciliatio ere term con lity through dictatorship by explaing the rontinuity through dictatorship by explaining pere resto eaceuld nocir num neor. Reir 2009. Remarkably for some, on the campaign, Mujica . 156). So that cone fiom the 1970..." (Churchill 2014 156). Some authors have tied the Tupamaros transition to electoral politics in the context of similar developments in Nicaragua and El Salvador. Churchill has f the dictatorship period as aberrant She the notion the Tupaman were always as much "Uruguaya" the Tupamaros were always as much "Uruguayn" as they were Cuba-hispired revolutionary Marxists. The unusual historical absence in Uruguay of powerful ecoronices This proved true not only of democratic politics, but of

the Tupamaros' unique ability to mix conflicting ideologies into praxis. In the early 1970s, many members of the Tupamaros had no qualms "expressing admira(2014, 159). Unlike 2014, 159). Unlike other leftist revolutionary groups of the era, the Tupamaros never rejected everything abou the backdrop to the widespread popularity acrossideologal boundaries of the Mujica presidency.

Also dismissive of the simplistic notion of dictatorship as a Cold War deviation, Federico Finchelstein's Ideological Origins offers a compelling and bold new answer to the question, was the military government fascist? In post dictatorship Argentine popular culUnited States, there was a strong tendersyip in the the regime with Nazism or fascism. to the dic with Nazism or fascin. That approach to the dictatorship drew, first, on a tendency among some to isolate the period as a a te - a horific interruption to modern, civil rule that must neve be pernitted again. In addition, it was based on the characterization of the in the international han ticularly in regard to the detention and torture of the Jewish joun rest own famous characterization of his ordeal as a descen nto a new Nazism.

Finchestin's linkage of dictatorship to fascism is more thoughtful and compelling. An aberrant, violent historical moment did not define the dictatorship as fascist, nor did the mistreatment of Timerman and other Jews in and of itself. The regime's fascist tendencies have their origins in longstanding Argentine ideological and political traditions extending back at least through the $1930 \mathrm{~s}$, and transcending both denocratic and dictatorial periods. This is especially evident in the construction of an internal, subversive enemy that concealed a foreign, intrusive presence. Draws 1978 FIFA World Cup of Soccer (held in Argentina), "the widely ace "the widely accepted fantasy that the enemy was not (Finchelstein 2014, 150). Reinforcing the notion the (Finchelstein 2014, 150). Reinforcing the notion that soccer was linked to the dictatorship and longstanding fascistic ideals of Argentine nationalism, the coach of junta learer and Cesar Luis Menotti thanked military junta leader and psychopath Admiral Emilio Massera the World Cup to Argentines as a defence of the rea Argentina against its enemies, where the dictatorsh dentified itself with the sacred and the hosting of the event as a victory over subversion.

Ideological Origins goes on to show another important and longstanding link between military dictato ship, long-teric fascistic inspirations fiom Europe. The central dictatorship objective of defending national sovereignty from Nazi Ge non al war drew from Nazi Germany and fascist Italy. All at once, in cin winnable, and could never really be over. This new identify en was a the subse War bene a value. The ferocity and absurdity War beca War bo the drew dir al on drew directlo on in Argentina. The latter had long held that dying for

Where Ideological Origins and Becoming

Where Ideological Origins and Becoming The Tupamaros dispense with dull binaries in trying to explain ditatorship not Justice in Argentina and Uruguy is preorition

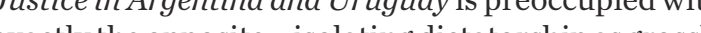
exactly the opp

The book asks, what is the relationship between transitional justice and memory? Francesca Less offers an unprecedented overview of theoretical an empirical literatures on amnesties, trials, truth commissions, reparations, and memory narratives. He Stern [ ] Stern [...] defined as "emblematic memory", a frameworkfor collectiveremembrance", that emerges from multiple human efforts, conflictual and competitive, to give meaning" to collective, decisive, or transforpublicor mipuling public or semipubic domains like the mass media, government events, street denonstrations and protests, consich constructed and selective, they are neither arbitrary inventions nor manipulations, but offer influential an convincing enunciations of often intensely disputed events (Lessa 2013, 20-21). Enruenatic or collective memory in Argentin

In a chapter on transitional justice in Argentin (still ongoing after more than three decades), Lessa is particularly taken with what she describes as the pection to the presidencyin 2003 This away fom the imply of the 1990 s and in away from the impunity of the $190 \mathrm{~s}$ and into a new period of prosections, as of Decer victions on the were 262 convictions on dictatorship-era crimes and counting. The jook correctly ties political change in 2003 and a new flen-poignant memializtion of milary otten-poignant memorialization of nilitary rule, from Pla a Plata estuary to the hundreds of memorial plaques rilling sites from which people were "dis

recalling sites from which people were "disappeared" Chapters on Uruguay trace asinilar uplifting story als collective, enblematic memies were pitted cals collective, enblematic memories were pitte gainst dictatorip impunity. Like Argentina, with he paste a finaljudicial a couste in the lal 1980s and 1990s "a momas as under a pall the late 1980s and 199s, a memory of silence and over the weed for truth, justice, repatriations, mem theed for truth, justice, repatriations, and As much as anything past (Lessa 2013, 218-219). As much as anything, Memory and Transitional Jusfine is a morality tale of two civil societies that have

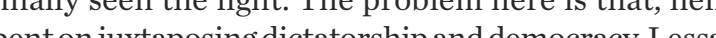
bent onjuxtaposing dictatorship and democracy, Less and memory, the runs with them for the length of and memory, then runs with them for the length of Carlos Menem for example, to the memory of silence and oblivion, like other critics of that administration (once sensibly described by a feral judge (once sensibly described by a federal judge as an illicit crime organization), Lessa assumes that the politics of broed politiolerm presidency was not a reflection in that the is till no s. Is that a credible assertion in that there is still no study of participatory politics period of denos whe period of democracy as open as that of the past fifteen years? Is it possible that what Lessa characterizes as silence and oblivion was, in fact, a reflection of an alternative memory, sense of justice, and criminal wron uring and after military rule?

Beginning with her arguments on emblematic memories, Lessa routinely assumes that there is a
collective memory on dictatorship in Argentina and 
Uruguay that has finally triumphed as a result of the current wave of prosecutions. There is simply no evicer to rection of political cultures in wite, middle-class urban enclaves in Argentina and of the Kirchner prestdences bur in Ras collective "Arsentine" memoris that a on cen "Ar fective for the white, urbaniddle class. Lessacites the 1090 imp ity instead of blaming the miltary and rov impunity, instead of blaming the military for the that narrative was overcome after 2003 (and blame squar "f laid at the feet of cher juctialaccountingand

Let's leave aside whether or not working Argentine living in the one million strong, largely impoverishe Forencio Varela municipality in Greater Bueno Aires ascribe or ascibed any blome for 1970 s politpare repre the poignant issue is the either-or binary of two demons

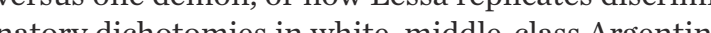
natory dichotomies in wite, midde-class Argentine pories Nobody heverconducted working people of colour in Vare and hundreds of working people of colour in Varela and hundreds of or " "appropriate" remembrance (that is to say Iessa's ch "appropriate" renies thance (hat is to say, Lessa's und and Uruguayans across class, race, and geographica bour don thave thecort."

While racialized class differences shape society throughout Latin America, unlike Guatemala and Chile, state terror in Argentina and Uruguay was not directe in the first instance at working people of color. In this not in symptathy with authoritarian rule, or especilly preoccupied with whether violence came from the dicpreoccupied withwhether violence came from the dictatorship or the guerrillas. Most remained distant from ith all regards and in a context where state violence was during the dictatorship, and in democracy afterward and without significant difference. In 2007, the world champion boxer Juan Martín Coggi remembered his riday evenings as a young teen-ager fighting in the

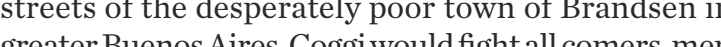

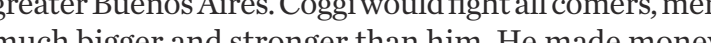
nuch bigger and stronger than him. He made money his improble victories is improbablevictor. One night in 1976, Coggi told me, people asked why. He noted hat everybodyknew that Falcon whet what

two hours and had covered the dictatorship period, this was the first and lives rence to military dictatorship touching the and millions of poor, working Arsentines of coggi, and nitions of poor, wertal to their lives bearing state teror was incidental to therr lives bearing in mind ong term continuities of poverty and police violence. Their memories and narratives of dictatorship what thely unk Most have never been asked whiddle recallof midde-class nemory-making institutions (often lionized by academics) that their memory "oblivion" is ignorance. For those who have yet to feel the impact of post-1983 transitional justice in their everyday lives, pro-human rights pro-human rights Argentina, president Raúl Alfonsin, died in 2009 . When newspapers reported on the work of his post-dictatorship human rights heroics, one Buenos Aires resident who had lived through intense pove funced at the She was evidenty stuck inobivion. She was evidently stuck in oblivion.

David M. K. Sheinin

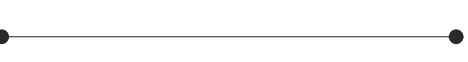

TO WAR. A JOURNEY ALONG THE FRONT OF WORLD WAR I

Arnout Hauben \& Johanna Spaey

Antwerpen: De Bezige Bij, 2013, 272 p.

nfotainment is supposed to disseminate historic knowledge to a broader audience, and I am fully aware that this unavoidably implies the loss of some nuance in favour of the genre's entertaining aspect. Th problem with Arnout Hauben and Johanna Spaey's

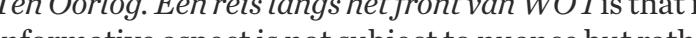

A few preliminary remarks. Reading the title raise some suspicions: the subtitle, A Journey Along the Fentline of World War 1 , niplies that the focus will be solely on the Western Front in Europe, neglecting the Easter Front, the clashes in Africa and Asia, and iven, at sea. It also defines the fronthe as a static given, whichis an exageration. Yes, the Western Front f 1914 and the year 1918, with both the Kaisersch and the year 1918, with both the Kaiserschlach

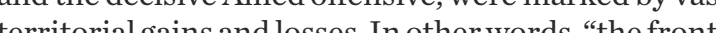
tern" is a bins and losses. In other words, "the fronten or a even, or especially, when ane Aside fon that, hisingsenthen tate to call the television programme a documentary and that the

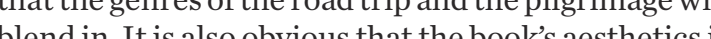
heavily infu als o heavily influenced, via the TV show, by the visual arts: the im a horiztate as the image of a horizon to depict a clear and complete overview. The question is, hhen, how these genres and aesthetics the theoks docume far claims. Luckily the authors are aware of their at once extensive and limited approach. They do signal that the Western Front was one of many war theatres (18) and that (Belgian) know of front and the campaigns in the Balkan fo the Italia front and the campaigns in the Balkans form an innovative inclusion for a Belgian target audience. The ers and of colonial troops, acknowledging that the war was not only fought by young white men. Hence, it does

It who makes explicit clams of causlity as to the infuence as Cana (56) and Italy (138), in the line of John as Canada (56) and Italy (138), in the line of John the Somme on post-war British society in The Face f Battle (Lon of Battle (London: Pimlico, 2004). As such, it touches con the relevance of the First World War and its con

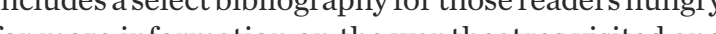
the individuals' lic stois mentioned.

Finally, the book is unsurprisingly reader-friendly and at times attempts to include an authentic and at thes attenpts to include an authentic documents, such as letters home and diary entries, documents, such as letters home and diary entries, to undermine the contemporary war propaganda by riving a voice to its victims, wishing to illustrate conflict's conting their tis the description of an elderly couple, wholost collecting WorldWar I hells a a kind of cly the shells may be seen as a "transeng egy. The shells may be seen as a "transgenerational doomed to fail. "Deberg mot is comed to fail. "De bergmoet immens zijn, net zo groo as huse f what the authors attempt on may try to conp of what the authors attempt: one may try to come to created, but ultimately the dead remain this past has

Yet this emotionally powerful insight is rendered ad absurdum through Hauben's exaggerated selfidentification with the soldiers on the frontlines. Empathy is a necessary attitude when undertaking draws between his but the paralls that Hauben mise ensène of their departure is telling a fanfare ise nlaying the thas post while the crew of thare band is playing the Last Post, while the crew of three kisse "zols "zoals elkejonge soldaat die in de Eerste Wereldoorlog "zoung soldier A

Another scene, in which Hauben draws a paralle between the artillery fire directed at Reims in 1914 metaphorical over that city anno 2013 , suggests the metapharcalcons the the 
expectations regarding the participatory nature of (the historical dimension is required.

However, this problematic attitude is in my opinion not the book's greatest flaw. Even more aggravating The the re the factual errors and contradictions. These are the byproduct of the attenpt to inustrate te feads to the usage of contradictory naps or picture

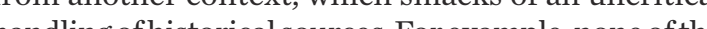
hor pex (lso, the depiction of the frontines east of Rims is Also, the depiction of the fronthines east of Reins is inexact: the map (78) indicates the trenches ran from

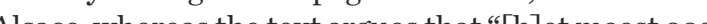
telijke deel van de Franse frontlinie wordt gevor

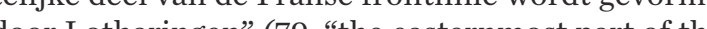
con rench frontline is shaped by Lorraine"). One may 1871 until 1918, yet this is not indicated on they from s7intilis ntioned in the text.

Another example of this impreciseness is the dicrion ofthe Belgian sector in the frontline around Diknuide, "in a B Somme, two years later. (32) Fingly, out le or somme, two years later. (32) Finally, on at least one Cermen touristsearwitness to the destruction of Germantoursts ba II to the destruction of Germany during World War II, supposedly by American Spitfires were British fighters, nat American bor po the Ger Bitish fighters, not Anerican on to the German survivors this makes no difference of course, but ou the authors. Evidently it would not be very tactful to confront the survivors with such errors in the minieflect on the ex how doct on the extensive use of eyewitness accounts in do Add some min mistakes, such

Add some minor mistakes, such as a translation error in Rudyard Kipling's My Boy Jack (71) and the mention of presumably lesser-known facts without providing background information (e.g., the presence his cons live up to its this book canctive up to its documentary claims. in documentary fiction, such as the oeuvres of W.G.

Sebald and Alexander Kluge - they even constitute the backbone of the authors' poetics. The problem with Spey and Hauben's narrative is, howe problen with Spaey and Hains aris the contradictions fiction but as a reportage. Hence, peal to the rions do not appear as an enlightening appeal to the reader's perspicacity. The book is rather miniseries. The this rese this respect, as is the pilgrimage. the crew of three pays homage to those fallen and those marred during and after the war and thereby continues the tradition of ough aly is of the wars cas sacrosancts.A thorough analysis of the war's causes and consequences is nearly completely absent, and the unprecedented brody nature of the war is blamed on the high-tech art of warfare - not mentioning the treatment of POW In spite of the inclusion of overs In spite of the inclusion of overseas troops and labourers, the (very few) Congolese troops on Belgian soil are - rather oddly - not mentioned, nor are whose descendants (also) live in Belgium Thermies,

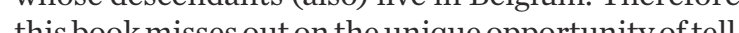
his book misses out on the unique opportunity of telling the war stories of Belgium's new citizens' ancestors Again, they are denied access to our (Belgian) practices ing to the all-soldier-are-sing them and by adherfail to reflect on their own which is an the which is a shame. The interest of a broader audience would the inve waned, since the books road-trip structhe various emotionally powown war theatres, and have offered the perfect counteru anechotes would have une remarks. Dry, because on a meta-lry and uneasy remarks. Dry, because on a meta-level; view view on World War I, and may hint at social debate

In general, this book had a lot of potential, but suffered from the authors' inaccuracy and uncritical position, which undermines the credibility of their work. flict but it also illustrates how easily such tor this conbect, but tialso ilustrates how easily such events have become persistent myths. Surely, a methodologically have roulted in a True and Final Story. Bus would no hould huled in a True and Final Story. But at least it world have led to a sound intering is, sadly frustration

\section{THE SLEEPWALKERS \\ HOW EUROPE WENT TO WAR IN 1914}

Christopher Clark

London: Allen Lane, 2012, 736 p.

014 is not just a year like any other: it carrie the commemorative weight of crisis, war, and eration, freedom and new beginning 2014 repsents the jemus $\mathrm{faced}$ character of the modern era here life-chang innovater of the whe life-changing innovations evoked alongside f three significat dates in of three significant dates in modern history brings hrough which abhorrentlessons whe wo live in today whin today came into being. This year, we commemorate ing of the American troops on the cost of Norm in of the Antican trops on the coast of Nornandy ine Wall 1989. All these dates matheor plic in public memory of contemporary Europe, and strongly iangle of 1914, 1944 and 1989, Europe was born as a triangle or 1914, 1944 and 1989, Europe was born as a reaction to the to to the bink destruction twice found oundations were laid to (soen. prosperous world we know today.

In 2012, historian Christopher Clark published a monumental work on the origins of World War Europe Went to War in 1914 ale Sleepwalkers - How Europe Went to War in 1914 already suggests that Clark ens to break with a tradion which holds an inportant spot within paradigmatic effort to name a culprit, to acuminate the ful and unmistak built Clark explicitly re dreadful and unmistakable guilt. Clark explicitly refuses to arrange the profusion of sources in a way which supports any teleological narrative. He writes: There the is work aims to retrace the steps which led to cerhis work aims to retrace the steps which led to cerquestions how the war broke out, not pin an questions how the war broke out, not primarily why. one decision affected the other. The result of this chain f reactions was - in its total scope and minacious out come - unrecognizable to the key players at the time. The Sleepwalkers closely examines the structures of power within Europe, poses questions of where thi actual power was executed and where not, and thu challenges the notion of "steadily building causal pressure" (xxvii) which led to an "inevitable" war

In his introduction, Christopher Clark starts with the time-and-again striking statement that it only took (hom the Saravo Archduke Franz Ferdinand and his wife Sophie, Duchess of Hohenburg, to the unleashing of an inferno that would extinct entire generations. Introducing his study with this statement might already raise awarenes withe reader that this rather awort time perod between the event and the outbreak could just be an excerpt, a detail from antterly more complex Clark describes what the historian encounters while claring to take un the his ying to take up the manifold argumentative threads hem into a cloth that lacks apologetic centh a curces, which oftes. Faced with an oversupply volid infor which often present an undersupply of valid information, Clark describes a "world war of

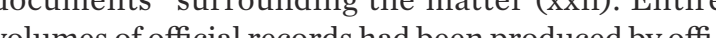
he war was ended and the treaty of Versilles signed. The was ended and the treaty of Versailles signed. these offial "ecords, however, are frequently chara derized by "endentious omissions" (xxii). Memoirs cloly oction" proved valuable the lections" proved valuable, the protagonists were often concerned with self-justification (xxii). It is these key the epenemeral, but rather obscure power structure their respective countries that Clark carefully revisits.

The Sleepwalkers is divided into threely The Sleepwalkers is divided into three parts. The oping argument will undertake in the following. Part One readjusts our "common knowledge" of the Part jevo assassination as being the point-of-origin of the first madern war. cirst no his wife were turned into stories, which to un a his wife were turned into stories, which took up a life nideon and witt be 
inherent political instability within the Balkan sphere which had been a reason for international confrontations for decades. Furthermore, he sheds a light on the political tensions which arose within the surrounding states. In this first part, Clark nakes it abundantly clear that the orighs of war are located within continuing iredentist discoun and our on the ticular. The latter was inform identity being grinded down within the multination commonwealthor Austria-Hungary. As Clark remarks, the $20^{\mathrm{t}}$ century and the Balkan wars of the 1990s have

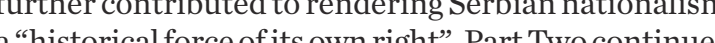
"histeres the development of Clarks argument by a dissecting of maps, allinces, and international relations. The and allianis and shows how they shifted throughout the out the development of the crisis. Indeed, the GermanAustrian Treaty, the Franco-Russian Alliance of 1894 the Entente Cordiale between France and Britain in (9) and the convention of ussia, are structures inoseally tions are only visible in retrospect. Part Three count down the days and recounts the last decisions (or decisons that whe nache to

as wh

As Thomas Laqueur ('Some Damn Fooling Thing', the title Theepwalker des the title The Sleepwalkers does not support the poin that Clark is making here. In his introduction, Clark that decisions mers that decissons months an "calculated steps" do neither constitute sleep). These "calculated steps" do neither constitute sleepwalking as far as my understanding of the concept goes, nor did Clarkmake any attempts to exonerate the protagonist Christopher Clark is a Christopher Clark is a master of his sources, balance with representing scen with representing scenes and episodes in an almost to torte manner. The narrative is constructed not to topple over a language that keeps the reader at scientific distance. The author balances the proportho ben thus deploying methodology - and the steady flow of his beautiful prose. Given that Clark's point is that he walkers, his narrative is saturated with agency of the historian. However, Clark touches upon the role of the historian only in passing. He acknowledges that access to First World War narratives and the conclusions that we contemporaries draw are a matter of viewpoint, social and scholarly contexts, and experiences we (re) make. A discussion of his role, that of the historian, as an architect of history in the making seems utterly the the master narrative of exclusive German guilt. That is in itself a very postmodern endeavor, though something which Clark is unaware of or does not conside it inilt is clat guilt is closely connected to Gernan self-awarenes indity identity were constructed as a reaction to this questhis. Clark mentions the Fischerschooland the effects this debate had on the reevaluation of Nazism and the Third Reich. Acommentary from the author on the disruptive, yet constructive potential of his work,

In his introduction, Clark already makes one of the most interesting points. He reframes the First World War as a "modern event". A modern crisis, in his terms, is characterized through the existence of "suicide thes shook the world during the 2000s. He goes on:

Behind the outrage of Sarajevo was an avowedly terroris organization with a cult of sacrifice, death and revenge, but this organization was extra-territorial, without clear geographical or political location, it was scattered in cells across political borders, it was unaccountable, its Inks to any sovereign government were oblique, hidden and certainly very difficult to discern from outside the organization. Indeed, one could even say that July 1914 (ins in the 1980s. (xxv)

Yes, modern warfare, if you may, is characterized through scattered groups which base their raison-d'être on rather conservative world views and rigid morals, pretending to defend their formative principles. Clark's nodern enemy has become abstract, impersonal, a sleeper or fanatic amidst us. Clark did find some and the suicide warriors of the $21^{\text {st }}$ century. first and formost, in my opinion, the July Crisiser, mod and forenost, in my opinion, the July Crisis is a the national concept. It heralded the end of the nation, for it carried the seed of supranational structures. A Clark has convincingly shown, the key decision makand forged to prevent intrusions into the respecive hand, forged to prevent intrisions into the respective allional sphere, and alliances on the other. As a consequence, the entire doxically posed a loming the th its Itwas chadoxically, posed aloon acterized through mutual distrust and an anbiguity Tha tin itelf mid lo That in itself might notbe a surprise or an extraordinary conclusion, but it already opens up a range of in particular with regard to contempory ofwar in particular with regard to contemporary controis a result of many of the aspects that Clion. Europe it is a supranany of the aspects that Clank reiterates; it is a supranational structire which seeks to bridge national interests and revi in the years proceding narrative that was absent in the years preceding the turou founding narrative has becof European founding narrative has becone more and Putin's opportunism hasled the European realm at the putin's opportunim "improbable brink or another "improbab " crisis. The Russiangovthe Ukraine and the EU might leat to a the Ukraine and the EU might lead to a furthering of powe ind to a strengtheng of perceived Eunculture, and to a strengthening of perceived Europea hegemony. As aconsequenc, Putyintervened andforcibly annexed the Crimea. The EU is clearly struggling

The rect sucess of anti-European poptis. The recent success of anti-European populis insts another dark clond over the pu project ing at an unsettling tendency to reiterate the concept of the issism. Whilst one may shar pean union, one aspect remains striking: although we might find ourselves in the era of many "posts" - postof the nation is by no means ded. The threat to sop the nation is by no me acing the altar of the European community became a mighty tre in thes being ready to restrain the influence the EU on hational sovereignty disturbingly reminds us of how he alla the weeks precens wing pelicy mingwere non-existing 1914, and that the policy making were non-existing in 1914, and that the int Eres the curopean crisis we encounter today, many of those hips between the European players are again osillatships between the European players are again oscillatinteres interests. Although the public opinion seems to remain against European intervention in the Ukrainian crisis, the conclusions that Christopher Clark has re the conclusions that Christopher Clark has reache alarmingly remind us of the reasons why Europe exist doing so.

Of course, the publication of The Sleepwalker predates the Ukrainian crisis. Nonetheless, one might Whant to pose these question to Christopher Clark: What are the implications of his work for the postWhat is the role of the historian in constron What is the role of the historian in constructing and Whtesting master narratives and collective identities? which were a direct result of the World Wars? 